\title{
Subclinical Celiac Disease Presented With Postpartum Low-Back Pain: Case report
}

\author{
Suleyman Baldane ${ }^{1}$, Suleyman Ipekci ${ }^{1}$, Fatih Sahin ${ }^{2}$, Emine Gul Baldane $^{3}$, \\ Pinar Karabagli ${ }^{4}$, Levent Kebapcilar ${ }^{1}$, Mustafa Kanat ${ }^{5}$ \\ ${ }^{1}$ Division of Endocrinology and Metabolism, Selcuk University Faculty of Medicine, Konya, Turkey \\ ${ }^{2}$ Department of Internal Medicine, Selcuk University Faculty of Medicine, Konya, Turkey \\ ${ }^{3}$ Department of Neurology, Konya Education and Research Hospital, Konya, Turkey \\ ${ }^{4}$ Department of Pathology, Selcuk University Faculty of Medicine, Konya, Turkey \\ 5 Division of Internal Medicine, Medipol University Faculty of Medicine, Istanbul, Turkey
}

\section{Introduction}

Malabsorption conditions (such as Celiac disease) and secondary causes of osteoporosis should be investigated in patients with pregnancy-associated osteoporosis (1)

Celiac disease (CD) is an autoimmune enteropathy commonly encountered in genetically predisposed individuals and precipitated by the ingestion of gluten-containing foods which results in malabsorption associated by immune mechanisms (2). In subclinical form of $C D$, there are serological findings and mucosal injury similar to the classical form but no gastrointestinal symptoms (3). Patients with subclinical CD may present with non-gastrointestinal findings such as iron deficiency anemia, teeth enamel coating abnormalities, high level of liver enzymes, and osteoporosis $(3,4)$. The development of osteoporosis may be accelerated in pregnant women with $C D$, due to the increased demand for calcium and increased bone resorption both observed during pregnancy. In this article, we present a case of subclinical CD presented with postpartum low-back pain, in whom we discovered severe lumbar vertebral osteoporosis.

\section{Case report}

A 35-year-old woman admitted to neurology clinic with severe low-back pain. Then she was referred to our endocrinology unit since diffuse vertebral signal loss of the lumbar vertebrae was detected in the magnetic resonance imaging (MRI) scan. The patient had given birth five months ago, and was breastfeeding. She was complaining of progressive postpartum low-back pain. Her pain was increasing with movement and decreasing with rest. There was no history of trauma, fever, night sweating, abdominal pain, diarrhea, nausea, vomiting or skin rashes. The patient was receiving no medications apart from the oral iron medication previously prescribed for iron deficiency anemia.

Upon physical examination, her height was $155 \mathrm{~cm}$, weight was $45 \mathrm{~kg}$, and body mass index was $18.7 \mathrm{~kg} / \mathrm{m}^{2}$; musculoskeletal examination revealed no tenderness in the back or hip joints with a full range of motion in both hip joints and neurological examinations was normal.

The laboratory analyses results were as follows: hemoglobin $10.2 \mathrm{~g} / \mathrm{dL}$ (normal: 12.0-16.0), serum calcium $7.2 \mathrm{mg} / \mathrm{dL}$ (normal: 8.4-10.2), phosphorus $2.0 \mathrm{mg} / \mathrm{dL}$ (normal: 2.3-4.7), ALP $605 \mathrm{U} / \mathrm{L}$ (normal: 40-150), gamma-glutamyl transferase $10 \mathrm{U} / \mathrm{L}$ (normal: 9-36), 25(OH) vitamin $D<5 \mathrm{ng} / \mathrm{mL}$ (normal: 30-150), and PTH $640 \mathrm{pg} / \mathrm{mL}$ (normal: 12-65. Thyroid, liver and renal function tests were within normal limits.

The patient's DXA (Lunar, Madison) scan revealed lumbar vertebral $\left(L_{1}-L_{4}\right)$ bone mineral density of $0.597 \mathrm{~g} / \mathrm{m}^{2}$ and $T$ and $Z$ score of -5.1 and -4.4 , respectively. Mild fracture (Grade 1 ) was observed $\mathrm{L}_{2}-\mathrm{L}_{3}-\mathrm{L}_{4}$ vertebrae in $\mathrm{X}$-ray; and hip MRI performed to eliminate any possibility of avascular necrosis revealed no pathological findings.

Serological tests were performed to investigate CD associated with malabsorption, in the patient who had severe osteoporosis, low calcium, hemoglobin, and vitamin D, and high PTH levels. Antigliadin IgG (1/40), anti endomisium IgA-IgG (respectively $1 / 40$ - and $1 / 20$ ) and tissue transglutaminase IgA-lgG (respectively high positive and low positive) tests were found to be positive. Esophagogastroduodenoscopy demonstrated mucosal hyperemia in the corpus and antral regions of the stomach. A lower duodenal biopsy showed moderate villous blunting and cript hyperplasia. A significant increase in lamina propria infiltration by lymphoplasmacytic and neutrophilic infiltration were observed. The number of intraepithelial lymphocytes per 100 epithelial cell was 36 (Figure 1,2).

The diagnosis of subclinical CD was made based on these findings and the patient was placed on a gluten-free diet, and calcium carbonate (1000 $\mathrm{mg}$, three times per day), and vitamin $\mathrm{D}_{3}$ (once $100.000 \mathrm{IU}$ intramuscular and $800 \mathrm{IU}$ one time per day oral drop) treatment. In addition, the patient was recommended to stop breastfeeding and she gave up breastfeeding before medical treatment.

At the $3^{\text {rd }}$ month follow-up visit, the patient was totally recovered from her low-back pain and follow-up blood tests showed hemoglobin level of $12.5 \mathrm{~g} / \mathrm{dL}$, serum calcium of $8.8 \mathrm{mg} / \mathrm{dL}$, phosphorus of $3.9 \mathrm{mg} / \mathrm{dL}$, ALP of $174 \mathrm{U} / \mathrm{L}$, vitamin D of $37.6 \mathrm{ng} / \mathrm{mL}$ and PTH of $52 \mathrm{pg} / \mathrm{mL}$. Bone mineral density measurement 6 months after initiation of therapy, using the same machine and read by the same radiologist, showed a significant increase at the lumbar vertebra (L1-L4); 1.072 $\mathrm{g} / \mathrm{m}^{2}$ and $\mathrm{T}$ and $\mathrm{Z}$ score -0.7 and -0.2 respectively.

\section{Discussion}

In the subclinical form of $C D$, there is limited involvement of the proximal portion of the small intestine. The distal portion is suggested to compensate for the lost functions of the proximal. Diagnosis of subclinical CD may be discovery incidentally or delays until occurring overt symptom of gastrointestinal system. Therefore, subclinical CD should be considered in patients with nonintestinal findings such as osteoporosis.

Osteoporosis is a well-known complication of CD. The prevalence of CD in osteoporotic individuals is approximately $3.4 \%$, whereas in non-osteoporotic individuals the prevalence is approximately $0.2 \%(5)$. The classical, subclinical and latent forms of CD all may play a role in the development of osteoporosis. Calcium and vitamin D deficiency, which relatively occurs secondary to malabsorption, contribute to the development of osteoporosis associated with CD. However, different mechanisms at the receptor level such as the RANK/RANK-L/osteoprotegerin system are currently known to play a role in the development of osteoporosis. The RANK-L is the final mediator of the cytokine (IL-1, TNF, IL-6) and hormone (such as PTH) network in bone. This soluble polypeptide protein belonging to the tumor necrosis factor ligand family is synthesized by osteoblast located in the cell membrane. The system is bound to its RANK receptor, expressed on cells of the osteoclastic line, and stimulates osteoclast maturation, while inhibiting apoptosis. Osteoprotegerin is an another component of this system, expressed by osteoblastic cells, inhibits bindings of RANKL/RANK system, maturation of osteoclast and resorbtion of bone. RANK-L is expressed by inflammatory and normal neutrophils, unlike OPG and RANK, which are expressed only by neutrophils exposed to an inflammatory processes. Therefore, neutrophils may contribute to activating RANKL/RANK system in subclinical CD (6). These mechanisms may explain osteoporosis in moderate forms of subclinical $C D$ and in latent $C D$ cases with no intestinal mucosa injury. On the other hand, the occurrence of early menopause and frequent amenorrhea in patients with CD may also contribute to the development of osteoporosis $(7,8)$.

Calcium absorption and urinary calcium excretion during pregnancy increase significantly, compared to the period before pregnancy, due to increased fetal calcium demand for fetal bone development. Bone resorption increases during pregnancy, an increase that has been demonstrated by histological and biochemistry tests (9).

Our case was an undiagnosed case of subclinical CD before pregnancy. She was most probably maintaining her bone metabolism without serious osteoporosis development due to the normal functioning of the non-involved distal segment of the intestine before pregnancy. However, hypocalcemia and severe vitamin $D$ deficiency developed as a result of increased calcium demand during pregnancy that was not adequately provided because of impaired intestinal absorption. Consequently, there was significant bone resorption associated with secondary hyperparathyroidism, with consequent development of severe lumbar osteoporosis that caused low-back pain. In conclusion, development of osteoporosis may be accelerated in patients with subclinical CD due to the increased calcium demand in pregnancy, hence patients become symptomatic. Subclinical CD should definitely be investigated in patients with postpartum osteoporosis. Moreover, the fact that dramatic improvement can be obtained in these patients through treatment with calcium and vitamin D should be kept in mind.
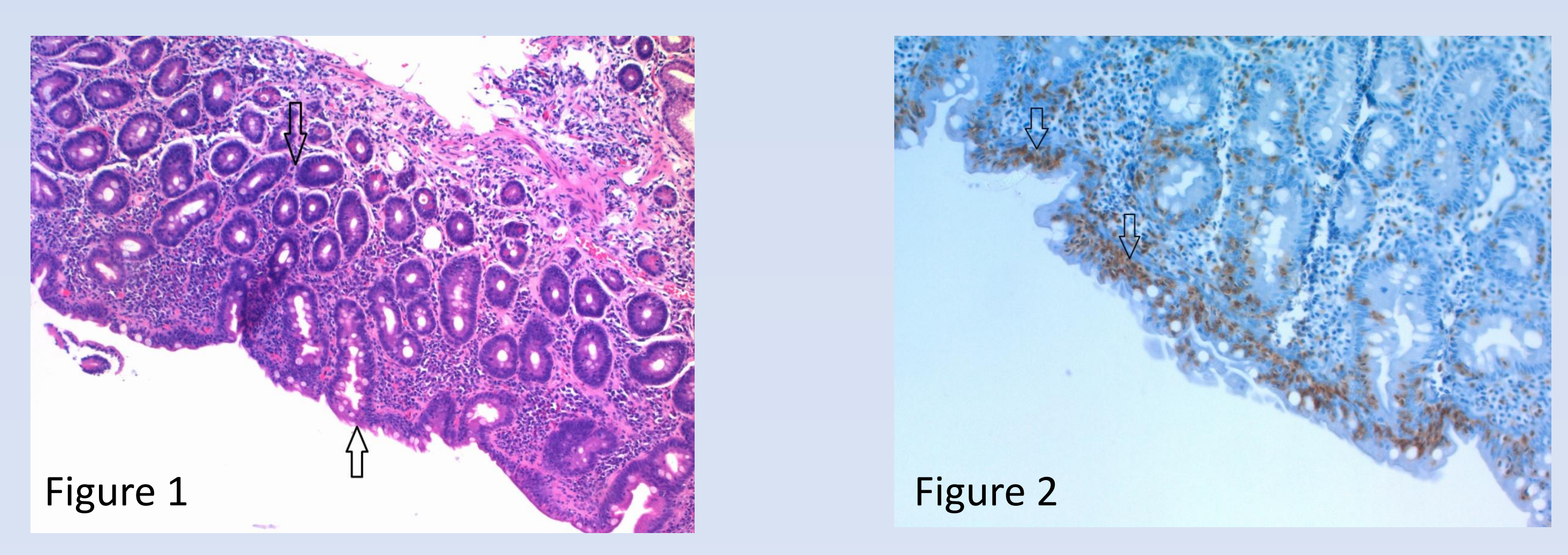

References

Di Gregorio S,

Green PH, Cellier C. Celiac disease. N Engl J Med 2007;357:1731-43.

3. Ludvigsson JF, Leffler DA, Bai IC, Biagi F, Fasano A, Green PH et al. The Oslo definitions for coeliac disease and related terms. Gut 2013;62:43-52. Tursi A, Giorgetti G, Brandimarte G, Rubino E, Lombardi D, Gasbarrini G. Prevalence
adults: an analysis on a 12-year observation. Hepatogastroenterology 2001;48:462-4.

Stenson WF, Newberry R, Lorenz R, Baldus C, Civitelli R. Increased prevalence of celiac disease and need for routine screening among patients with
osteoporosis. Arch Intern Med $2005 ; 165: 393-9$ osteoporosis. Arch Intern Med 2005;165:393-9.

Bianchi ML, Bardella MT. Bone in celiac disease. Osteoporos Int 2008;19:1705-16

Molteni N, Bardella MT, Bianchi PA. Obstetric and gynecological problems in women with untreated celiac sprue. J Clin Gastroenterol 1990;12:37-9. Gallagher JC. Effect of early menopause on bone mineral density and fractures. Menopause 2007;14:567-71. 